\title{
IMPEDANCE SPECTROSCOPY STUDY OF BIS(2-AMINO-6-METHYLPYRIDINIUM) TETRACHLORIDOZINCATE
}

\author{
A. OUESLATI* ${ }^{*}$ M. HAMDI ${ }^{\dagger}$, I. CHAABANE ${ }^{*}$, \\ F. HLEL ${ }^{\S}$ and M. GARGOURI ${ }^{\S}$ \\ Laboratoire de l'état solide \\ Faculté des Sciences de Sfax, B. P. 802, 3018 Sfax, Tunisie \\ *oueslatiabderrazek@yahoo.fr \\ †hamdymed@gmail.com \\ *chaabane.iskandar@gmail.com \\ \$faouzihlel@yahoo.fr \\ "mohamed.gargouri@fss.rnu.tn \\ Received 14 June 2012 \\ Revised 12 September 2012 \\ Accepted 22 October 2012 \\ Published 16 January 2013
}

\begin{abstract}
The complex impedance of the bis(2-amino-6-methylpyridine) tetrachloridozincate compound $\left(\mathrm{C}_{6} \mathrm{H}_{9} \mathrm{~N}_{2}\right)_{2} \mathrm{ZnCl}_{4}$ has been investigated in the temperature range $313-403 \mathrm{~K}$ and in the frequency range $200 \mathrm{~Hz}-5 \mathrm{MHz}$. The impedance plots show semicircle arcs at different temperatures and an electrical equivalent circuit has been proposed to explain the impedance results. The circuits consist of the parallel combination of bulk resistance $R_{p}$ and constant phase elements (CPE). The bulk resistance of the material decreases with rise in temperature. dc conduction activation energies are estimated from Arrhenius plots. The frequency-dependent conductivity data are fitted in the modified power law: $\sigma_{\mathrm{ac}}(\omega)=\sigma_{\mathrm{dc}}+A_{1} \omega^{s 1}+A_{2} \omega^{s 2}$. Dielectric data were analyzed using complex electrical modulus $M^{*}$ at various temperatures. The modulus plot can be characterized by full width at half height or by $\beta$ values of Kohlrausch-William-Watts (KWW) function. Activation energy of hopping is almost close to the activation energy of conduction suggesting a hopping transport mechanism.
\end{abstract}

Keywords: Inorganic-organic hybrid; electrical properties; equivalent circuit; modulus formalism; ionic conductivities.

\section{Introduction}

Considerable interest has been devoted to compounds of the general formula $\mathrm{A}_{2} \mathrm{MX}_{4}$ where $\mathrm{A}$ is an organic cation, $\mathrm{M}$ a divalent metal and $\mathrm{X}$ a halogen $(\mathrm{Cl}, \mathrm{Br}) .^{1,2}$ Organic-inorganic hybrid metal halides have received extensive attention in recent years owing to their great fundamental and practical interest. ${ }^{3,4}$ This is because of their special magnetic,$^{5}$ electronic ${ }^{6}$ and optoelectronic properties. $^{7}$ The influence of the features of the organic

${ }^{*}$ Corresponding author. 


\section{A. Oueslati et al.}

cations on the packing interactions that govern the crystal organization is expected to affect the packing and then the specific properties.

Recently, much concern has been shown in the synthesis of low-dimensional crystalline inorganic-organic metal halides with a possibility of incorporating properties associated with functional inorganic and organic moieties. A group of crystals, containing heteroaromatic cations like: pyridinium, substituted pyridinium and imidazolium ones, have been recently synthesized and characterized. ${ }^{11-13}$ Since aromatic heterocyclic cations are bestowed a significant electric dipole moment, thus some halogenometallates containing these cations form strongly polar structures. The presence of polar properties in the materials described above is usually a result of the organic cations dynamics, which play a key role in the induction of ferroelectricity. The results, obtained by us for the group of ionic compounds containing aromatic cations, trace a direction of search for the novel polar materials.

In this work we present the results of the impedance spectroscopy, the temperature and frequency dependence of the electrical conductivity and the modulus properties of the bis(2-amino-6-methylpyridine) tetrachloridozincate compound. ${ }^{13}$

\section{Experimental Procedure}

The bis(2-amino-6-methylpyridine) hexachloridozincate compound $\left(\mathrm{C}_{6} \mathrm{H}_{9} \mathrm{~N}_{2}\right)_{2} \mathrm{ZnCl}_{4}$ was synthesized by mixing $\left[\mathrm{C}_{6} \mathrm{H}_{8} \mathrm{~N}_{2}\right]$ (purity $99 \%$; FLUKA) and $\mathrm{ZnCl}_{2}$ (purity 98\%; FLUKA) in $\mathrm{HCl}$ (2M) aqueous solution in molar ratio 1:1 according to the reaction.

$$
2\left[\mathrm{C}_{6} \mathrm{H}_{8} \mathrm{~N}_{2}\right]+\mathrm{ZnCl}_{2} \stackrel{(2 \mathrm{M}) \mathrm{HCL}}{\longrightarrow}\left(\mathrm{C}_{6} \mathrm{H}_{9} \mathrm{~N}_{2}\right)_{2} \mathrm{ZnCl}_{4} \text {. }
$$

After few days, crystalline samples were obtained by slow evaporation at room temperature. X-ray powder diffraction pattern was recorded using a Philips PW 1710 diffractometer operating with copper radiation $\lambda_{K \alpha}=1.5418 \AA$. Unit cell parameter of the synthesized compound was refined by the least square method.

The differential scanning calorimetry was performed using a NETZSCH DSC 204 Phoenix instrument using aluminum capsules. The DSC measurement was performed from 285 upto $473 \mathrm{~K}$ at an average heating of $5 \mathrm{~K} / \mathrm{min}$ with a sample weight $15.12 \mathrm{mg}$.

The impedance spectroscopy was performed on pellet discs of about $8 \mathrm{~mm}$ diameter and about
$1.1 \mathrm{~mm}$ in thickness. Electrical impedances were measured in the frequency range from $200 \mathrm{~Hz}$ to $5 \mathrm{MHz}$ and the temperature range between 313 and $398 \mathrm{~K}$ with the TEGAM 3550 ALF automatic bridge monitored by a microcomputer.

Complex impedance data, $Z^{*}$, can be represented by its real, $Z^{\prime}$, and imaginary, $Z^{\prime \prime}$, parts by the relation $Z^{*}=Z^{\prime}-i Z^{\prime \prime}$. The real and the imaginary parts of the electrical modulus are:

$$
M^{\prime}=\omega C_{0} Z^{\prime \prime}, \quad M^{\prime \prime}=\omega C_{0} Z^{\prime},
$$

with $C_{0}=\varepsilon_{0} S / e$, where $\varepsilon_{0}$ is the permittivity of the free space, $S$ and $e$ are the area and the thickness of the pellet, respectively, and $\omega=2 \Pi f, f$ being the frequency in hertz.

\section{Results and Discussion}

\subsection{X-ray powder analysis}

The X-ray powder diffractogram (Fig. 1) reveals that the synthesized compound crystallizes in the triclinic system with $P \overline{1}$ space group, and the refined unit cell parameters are: $a=7.65(3) \AA, \quad b=$ 7.93(4) $\AA, c=15.63(3) \AA, \alpha=81.12(2)^{\circ}, \beta=79.26$ $(2)^{\circ}$ and $\gamma=90.15(7)^{\circ}$ which are in good agreement with the literature values. ${ }^{13}$

\subsection{Calorimetric study}

The results of the calorimetric study of bis(2-amino6-methylpyridine) tetrachloridozincate as presented in Fig. 2 show one endothermic peak at $455 \mathrm{~K}$. The values of enthalpy and entropy of this melting point were $\Delta H=77.25 \mathrm{~kJ} \mathrm{~mol}^{-1}$ and $\Delta S=$ $170.25 \mathrm{JK}^{-1} \mathrm{~mol}^{-1}$, respectively.

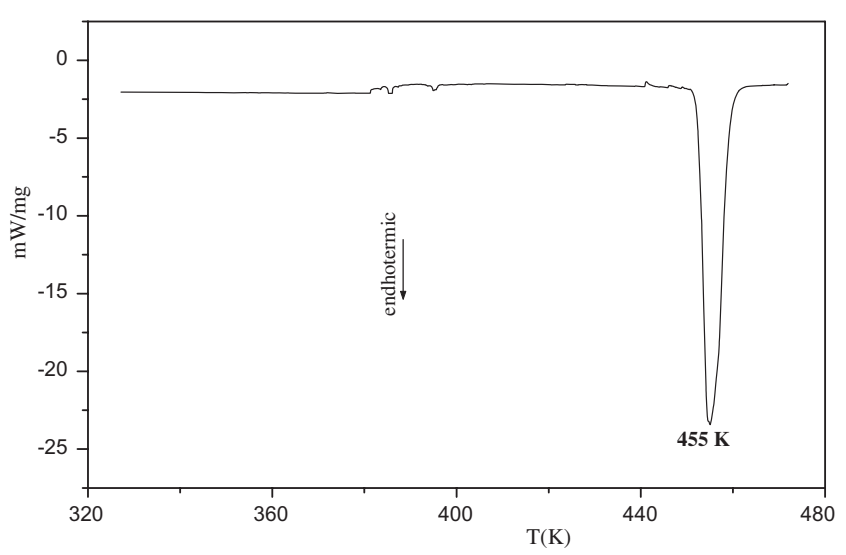

Fig. 1. Differential scanning calorimetry. 


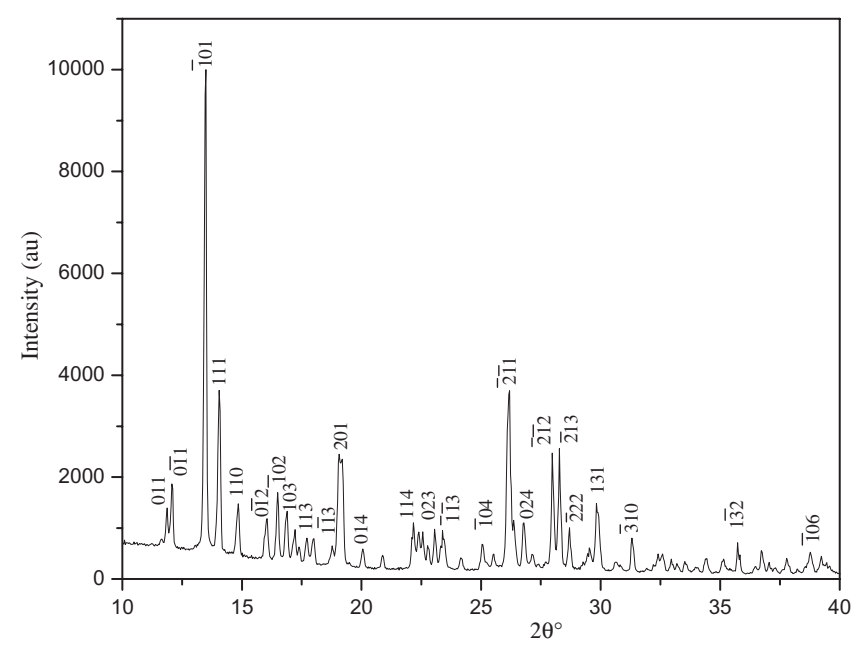

Fig. 2. X-ray powder diffraction pattern of bis(2-amino-6methylpyridine) tetrachloridozincate at room temperatures.

\subsection{Electrical impedance analysis}

Impedance spectroscopy is a useful method to resolve the contributions of various processes such as bulk, grain boundary and electrode effect in the specified frequency domain. In addition, the resistance and the capacitance associated with the solids could be estimated using impedance spectroscopy.

Figures 3(a) and 3(b) show the imaginary impedance $Z^{\prime \prime}$ versus the real impedance $Z^{\prime}$ of bis(2-amino6-methylpyridine) tetrachloridozincate sample at several temperatures (from $200 \mathrm{~Hz}$ to $5 \mathrm{MHz}$ ). Similar results have been observed for other samples and in all cases the experimental complex impedance plot comprises a single semicircle. The experimental arcs are found to be depressed semicircles with their

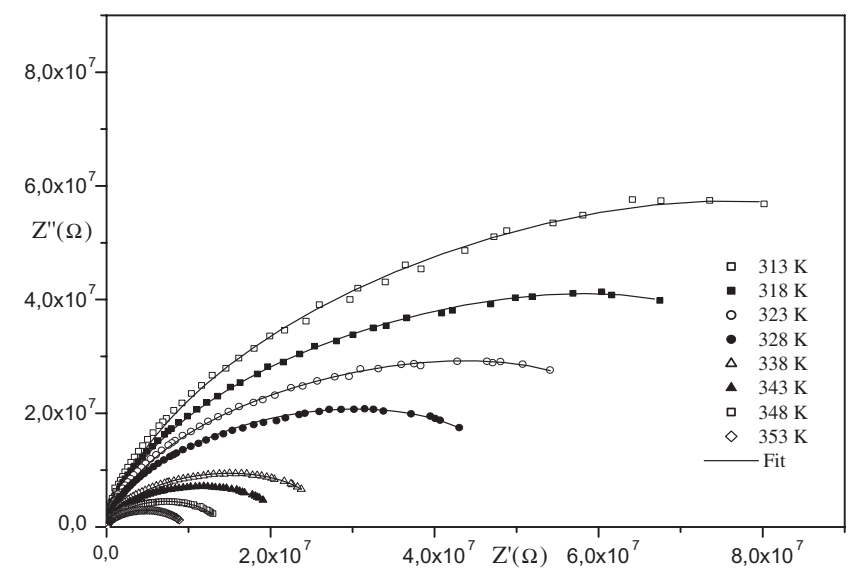

(a) centers below the real axis rather than on the real axis. The angle, by which such a semicircular arc is depressed below the real axis, is related to the width of the relaxation time distribution. As temperature increases, the radius of the arc corresponding to the bulk resistance of the sample decreases indicating an activated conduction mechanism. ${ }^{14,15}$ These features indicate that the relaxation time $\tau$ is not single valued but distributed continuously or discretely around a mean, $\tau_{m} \omega_{m}=1 .^{16}$

The complex impedance spectrum from Fig. 3 can be interpreted by means of equivalent circuit. Indeed, the corresponding equivalent circuit presented in Fig. 4 is essentially made up of a parallel combination of resistance $R p$ (polarization resistance) with a constant phase element CPE (capacity of the fractal interface) of the sample. Moreover, the impedance of CPE is given by:

$$
Z_{\mathrm{CPE}}=\frac{1}{Q(j \omega)^{\alpha}}
$$

where $Q$ indicates the value of capacitance of the $\mathrm{CPE}$ element and $\alpha$ is the fractal exponent: if $\alpha=1$, the element is an ideal capacitor, if $\alpha=0$, it behaves as a frequency-independent ohmic resistor. $j$ and $\omega$ represent imaginary unit and angular frequency, respectively.

The real $\left(Z^{\prime}\right)$ and imaginary $\left(-Z^{\prime \prime}\right)$ components of the whole impedance of this circuit were calculated according to the following expressions:

$$
\begin{array}{r}
Z^{\prime}=\frac{R_{P}^{2} Q \omega^{\alpha} \cos (\alpha \pi / 2)+R_{P}}{\left(1+R_{P} Q \omega^{\alpha} \cos (\alpha \pi / 2)\right)^{2}} \\
+\left(R_{P} Q \omega^{\alpha} \sin (\alpha \pi / 2)\right)^{2}
\end{array}
$$

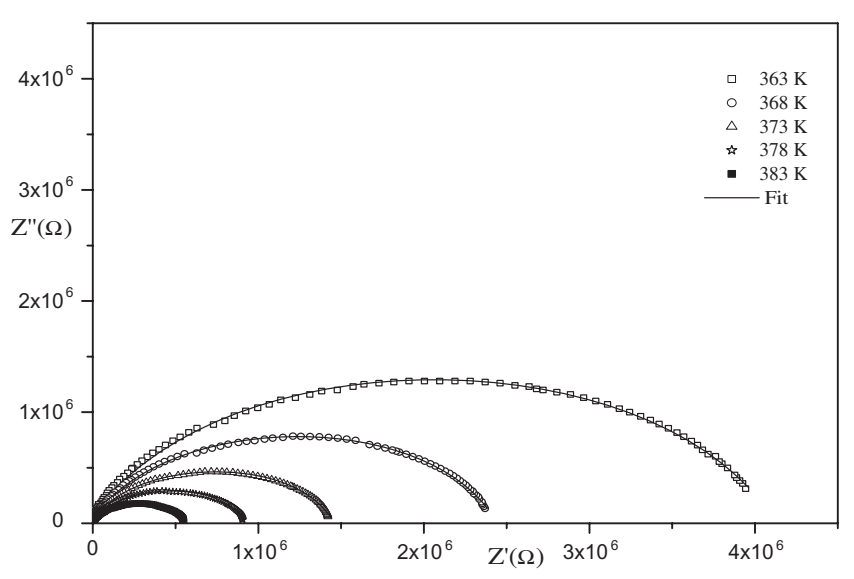

(b)

Fig. 3. Cole-Cole plots for bis(2-amino-6-methylpyridine) tetrachloridozincate at different temperatures. 


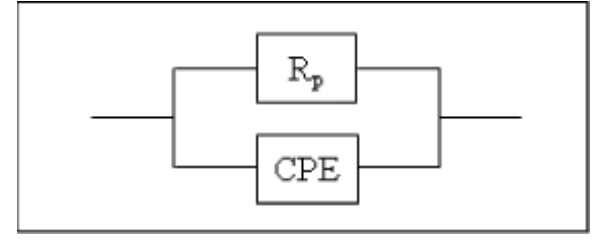

Fig. 4. Equivalent circuit model.

$$
-Z^{\prime \prime}=\frac{R_{P}^{2} Q \omega^{\alpha} \sin (\alpha \pi / 2)}{\left(1+R_{P} Q \omega^{\alpha} \cos (\alpha \pi / 2)\right)^{2}} .
$$

The resistance $R_{p}, Q$ and $\alpha$ have been simulated using a mean square method which consists to minimize the difference between the experimental and calculated data.

The fitted values of different parameters are listed in Table 1. It is obvious that all the capacitance values $Q$ are in the range of $p F$. This implies that the semi-circulars observed at several temperatures are essentially the response of grain bulk interior. The $\alpha$ values vary in $0.64-0.84$ range, indicating the weakness interaction between the localized sites. ${ }^{17}$

The electrical conductivity $\sigma_{p}$ was obtained from $\left(R_{p}\right)$ by means of the relation:

$$
\sigma_{p}=\frac{e}{R_{p} S},
$$

Table 1. Extracted parameters for the circuit elements of the $\mathrm{Zn}$ (II) with 2-amino-6-methylpyridine compound, where $Q$ indicates the value of capacitance of the CPE element, $\alpha$ is the fractal exponent and $R p$ is the polarization resistance.

\begin{tabular}{lccc}
\hline $\mathrm{T}(\mathrm{K})$ & $R_{p}(\Omega)$ & $\mathrm{Q}(\mathrm{F})$ & $\alpha$ \\
\hline 313 & $8.45 \mathrm{E}+07$ & $1.02 \mathrm{E}-12$ & 0.841 \\
318 & $7.62 \mathrm{E}+07$ & $1.44 \mathrm{E}-12$ & 0.821 \\
323 & $7.10 \mathrm{E}+07$ & $1.83 \mathrm{E}-12$ & 0.803 \\
328 & $5.42 \mathrm{E}+07$ & $2.36 \mathrm{E}-12$ & 0.781 \\
333 & $5.08 \mathrm{E}+07$ & $2.37 \mathrm{E}-12$ & 0.765 \\
338 & $2.81 \mathrm{E}+07$ & $4.18 \mathrm{E}-12$ & 0.751 \\
343 & $2.22 \mathrm{E}+07$ & $5.14 \mathrm{E}-12$ & 0.732 \\
348 & $1.47 \mathrm{E}+07$ & $7.06 \mathrm{E}-12$ & 0.721 \\
353 & $9.72 \mathrm{E}+06$ & $8.29 \mathrm{E}-12$ & 0.712 \\
358 & $9.13 \mathrm{E}+06$ & $9.85 \mathrm{E}-12$ & 0.705 \\
363 & $4.21 \mathrm{E}+06$ & $12.1 \mathrm{E}-12$ & 0.701 \\
368 & $2.50 \mathrm{E}+06$ & $13.7 \mathrm{E}-12$ & 0.696 \\
373 & $1.50 \mathrm{E}+06$ & $16.7 \mathrm{E}-12$ & 0.691 \\
378 & $9.55 \mathrm{E}+05$ & $19.4 \mathrm{E}-12$ & 0.686 \\
383 & $5.81 \mathrm{E}+05$ & $22.1 \mathrm{E}-12$ & 0.681 \\
388 & $3.90 \mathrm{E}+05$ & $26.2 \mathrm{E}-12$ & 0.669 \\
393 & $2.88 \mathrm{E}+05$ & $28.9 \mathrm{E}-12$ & 0.662 \\
398 & $2.61 \mathrm{E}+05$ & $33.7 \mathrm{E}-12$ & 0.643 \\
\hline
\end{tabular}

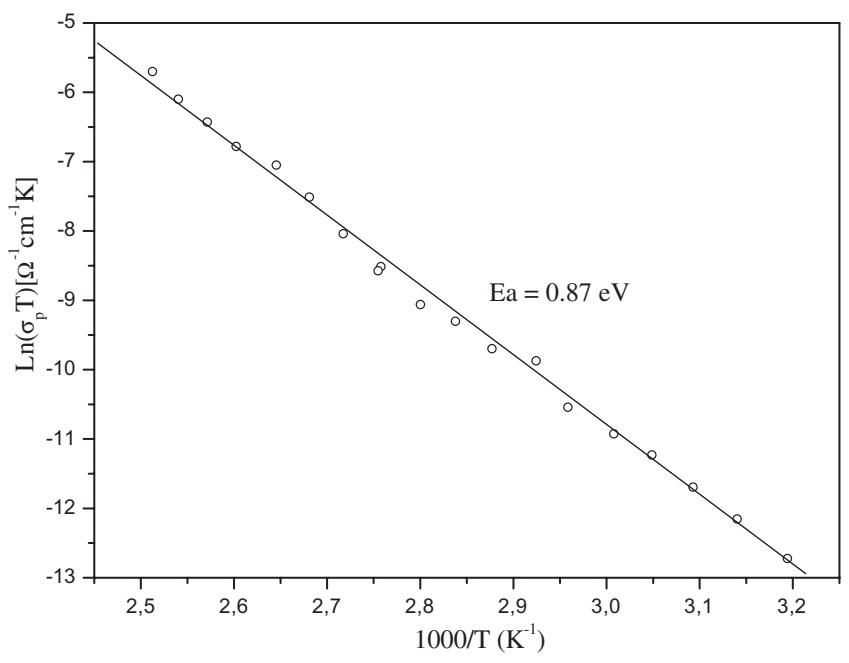

Fig. 5. Dependence of $\operatorname{Ln}\left(\sigma_{p} T\right)$ on temperature for bis $(2-$ amino-6-methylpyridine) tetrachloridozincate.

where $(e / S)$ represents the sample geometrical ratio. The temperature dependence of the electrical conductivity is represented in Fig. 5 in the form of $\ln \left(\sigma_{p} T\right)$ versus $1000 / T$. An Arrhenius type behavior, $\sigma_{p} T=B \cdot \exp \left(-E_{a} / k_{\beta} T\right)$, is shown. Following the Arrhenius law, the obtained activation energy is $E_{a}=0.87 \mathrm{eV}$. The enhanced conduction characteristics have been attributed to the increase of space charge conduction. ${ }^{18}$

Figure 6(a) shows the frequency dependence of $Z^{\prime}$ and $Z^{\prime \prime}$ at $373 \mathrm{~K}$. As the frequency increases, $Z^{\prime \prime}$ increases, whereas $Z^{\prime}$ decreases. This trend continues up to a particular frequency which in $Z^{\prime \prime}$ occupies a maximum value and in $Z^{\prime}$ intersects. Furthermore, if the frequency increases both $Z^{\prime}$ and

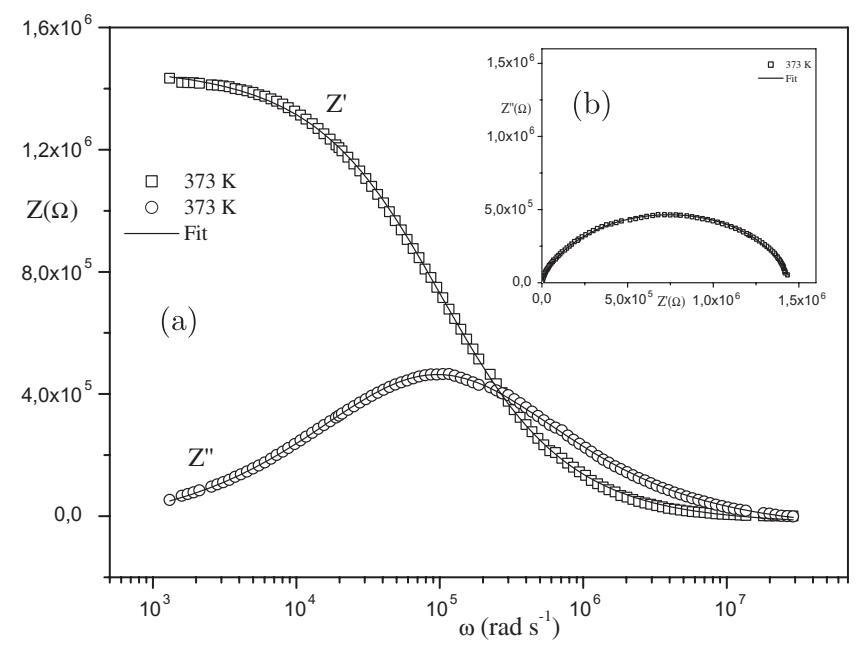

Fig. 6. Frequency dependence of $Z^{\prime}$ and $Z^{\prime \prime}$ and (b) corresponding to Argand diagram (inset). 
$Z^{\prime \prime}$ decrease, and above $10^{6} \mathrm{~Hz}$ both values merge with $X$-axis. This indicates that there exists a relaxation phenomenon. ${ }^{19}$ Figure $6(\mathrm{~b})$ shows that Argand diagram (imaginary part of complex impedance $Z^{*}$ versus its real part) allows the determination of bulk ohmic resistance as a function of temperature and thus temperature dependence of conductivity. ${ }^{20}$ The good conformity of the calculated lines with experimental data observed in Fig. 6(a) indicates that the suggested equivalent circuit describes the crystal-electrolyte interface reasonably well.

Figure 7 shows the normalized imaginary part of impedance $\left(Z^{\prime \prime} / Z_{\max }^{\prime \prime}\right)$ as a function of frequency in the material at several temperatures. The $Z^{\prime \prime} / Z_{\max }^{\prime \prime}$ parameter exhibits a peak in slightly asymmetric degree at each temperature especially at high temperatures. At the peak, the relaxation is defined by the condition, $2 \pi f_{p} \tau=1$, where $\tau$ is the relaxation time.

The relaxation frequency in Fig. 8 obeys the Arrhenius relation given by:

$$
\omega_{p}=\omega_{0} \exp \left(-\frac{E_{f}}{K_{\beta} T}\right)
$$

where $\omega_{0}$ and $E_{f}$ are pre-exponential factor and activation energy, respectively. The activation energy corresponding to non-Debye type of relaxation has been calculated to be $0.84 \mathrm{eV}$, which is almost equal to the value of activation energy calculated from temperature-dependent conductivity studies.

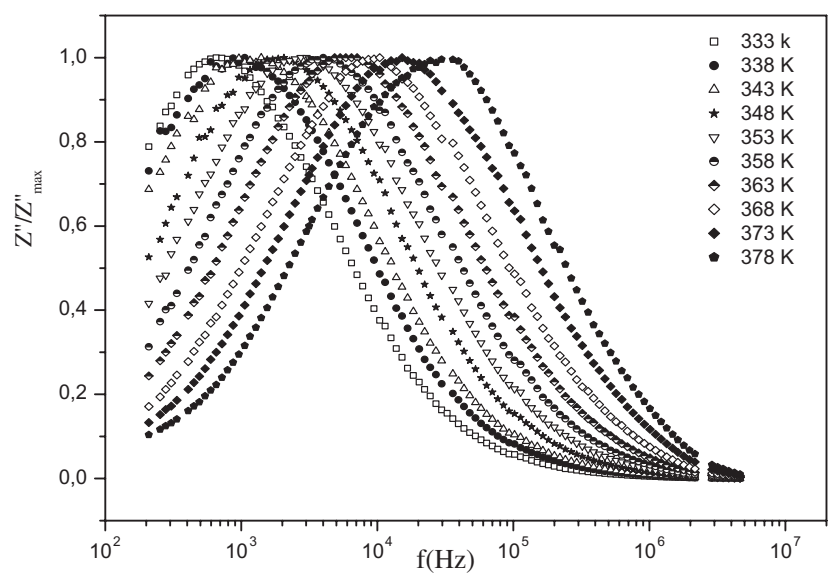

Fig. 7. Normalized imaginary parts, $Z^{\prime \prime} / Z_{\max }^{\prime \prime}$ of impedance as a function of frequency.

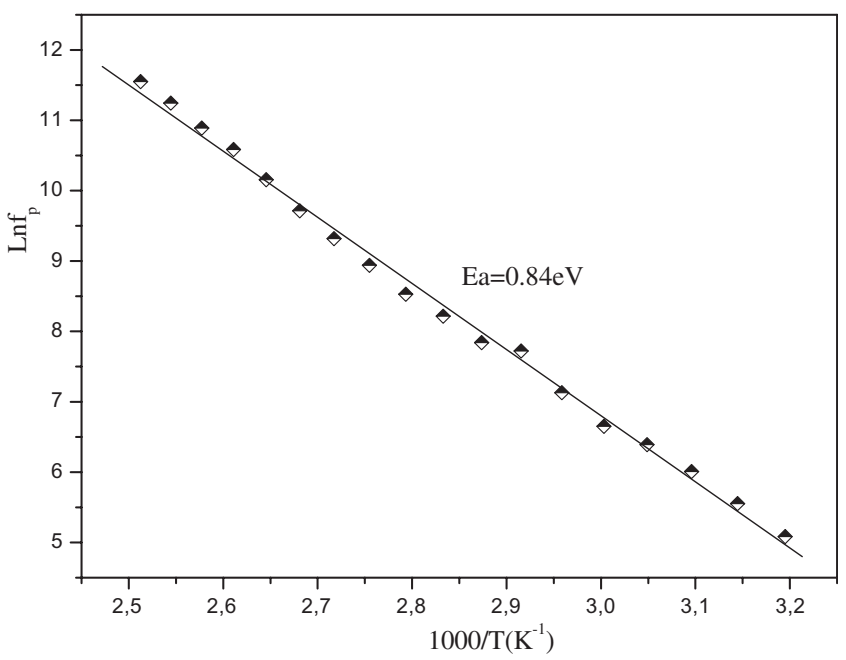

Fig. 8. Temperature dependence of the relaxation frequency $f_{p}$.

\section{4. dc and ac electrical conductivity}

The origin of frequency dependence of conductivity in the relaxation phenomenon arises due to mobile charge carriers. The ac conductivity $\left(\sigma_{\mathrm{ac}}\right)$ was calculated using the relation:

$$
\sigma_{\mathrm{ac}}=\frac{e}{S} \frac{\sin \theta}{|Z|}
$$

where $e$ is the sample thickness, $S$ is the cross-sectional area of the electrode deposited on the sample, $|Z|$ is the complex impedance module and $\theta$ is the phase angle.

The angular frequency dependence of ac conductivity at various temperatures for the sample is shown in Fig. 9. The conductivity has dispersion at all frequencies of the sample. The phenomenon of

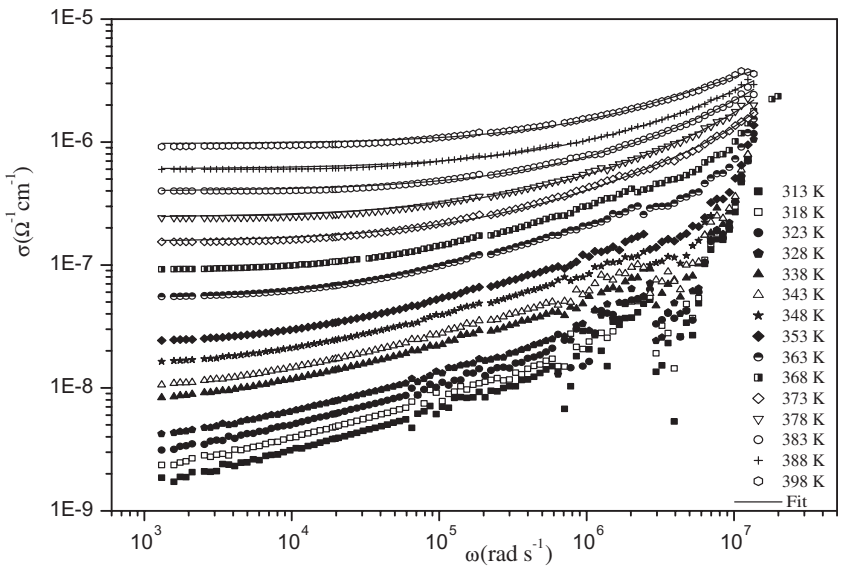

Fig. 9. Frequency dependence of ac conductivity of bis(2amino-6-methylpyridine) tetrachloridozincate at different temperatures. 


\section{A. Oueslati et al.}

the conductivity dispersion is generally analyzed using Jonscher's law ${ }^{21-23}$ :

$$
\sigma_{\mathrm{ac}}(\omega)=\sigma_{\mathrm{dc}}+A \omega^{s},
$$

where $\sigma_{\mathrm{dc}}$ is the direct current conductivity of the sample, $A$ constant for a particular temperature and $s(0<s<1)$ is the power exponent. The power law has been applied to many materials such as glasses and amorphous semiconductors. ${ }^{24-28}$

$$
\sigma_{\mathrm{ac}}(\omega)=\sigma_{\mathrm{dc}}+A_{1} \omega^{s 1}+A_{2} \omega^{s 2}
$$

which matched well with our data. $A_{1}$ and $A_{2}$ coefficients and $s 1$ and $s 2$ are critical exponents in different frequency regions. The first ac term, dominating at low frequencies, corresponds to translational hopping motion $(0<s 1<1)$. The second ac term dominating at high frequencies corresponds to well-localized hopping and/or reorientational motion and has values in the range $1<s 2<2 .^{29,30}$

This model is based on ionic hopping in ionically conducting crystals. It assumes adiabatic hops associated with retarded response of the "surroundings" (e.g., motion of neighboring mobile ions or local motion of the anionic framework) to such a hop. The retarded response imparts a time dependence to the barrier height for a backward hop from the new ion position leading to correlated forward backward hops and a concomitant dispersive frequency-dependent conductivity.

dc conductivity data are plotted in Arrhenius format as $\ln \left(\sigma_{\mathrm{dc}}\right)$ versus $1000 / T$ (Fig. 10), and all shown Arrhenius-type behavior described by:

$$
\sigma_{\mathrm{dc}}=A \exp \left(-\frac{E_{\sigma}}{K T}\right)
$$

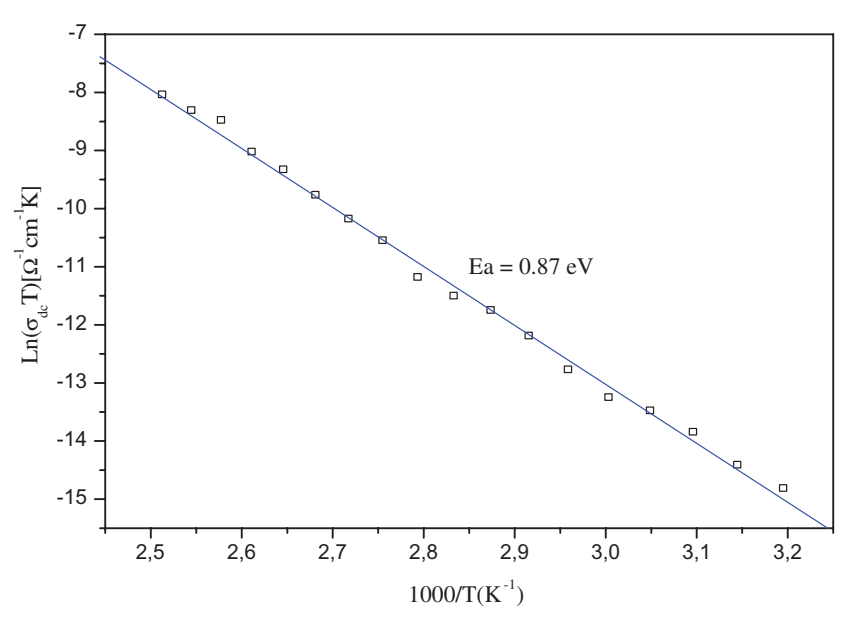

Fig. 10. Temperature dependence of $\ln \left(\sigma_{\mathrm{dc}} T\right)$. where $A$ is the pre-exponential factor. The calculated activation energy in bis(2-amino-6-methylpyridine) hexachloridozincate is $0.87 \mathrm{eV}$.

\subsection{Modulus studies}

The electrical modulus formalism is an important theory formulated by Macedo et al. ${ }^{31,32}$ This formalism permits to study charge transport processes such as mechanism of electrical transport, conductivity relaxation and ion dynamics as a function of frequency and temperature in ion conductors. It also suppresses the effect of the electrode polarization and/or mobile ion polarization, and highlights the smallest capacitance measured. The complex electric modulus $\left(M^{*}\right)$ can be represented by the following equation ${ }^{33-35}$ :

$$
\begin{aligned}
M^{*} & =1 / \varepsilon^{*}=M^{\prime}+j M^{\prime \prime}=j \omega C_{0} Z^{*} \\
& =M_{\infty}\left[1-\int_{0}^{\infty} e^{-j \omega t}\left(-\frac{d \phi(t)}{d t}\right) d t\right],
\end{aligned}
$$

where $M^{\prime}$ and $M^{\prime \prime}$ are the real and imaginary part of the complex modulus $M^{*}, M_{\infty}$ is the high frequency value of $M^{\prime}$. The function $\Phi(t)$ gives the time evolution of the electric field with in the dielectric.

Figure 11 shows the angular frequency dependence of the imaginary part of the electric modulus of bis(2-amino-6-methylpyridine) hexachloridozincate at several temperatures. The asymmetric modulus peaks shifts towards higher frequency side indicating correlation between motions of mobile ion charges. ${ }^{36,37}$ The asymmetry in peak broadening

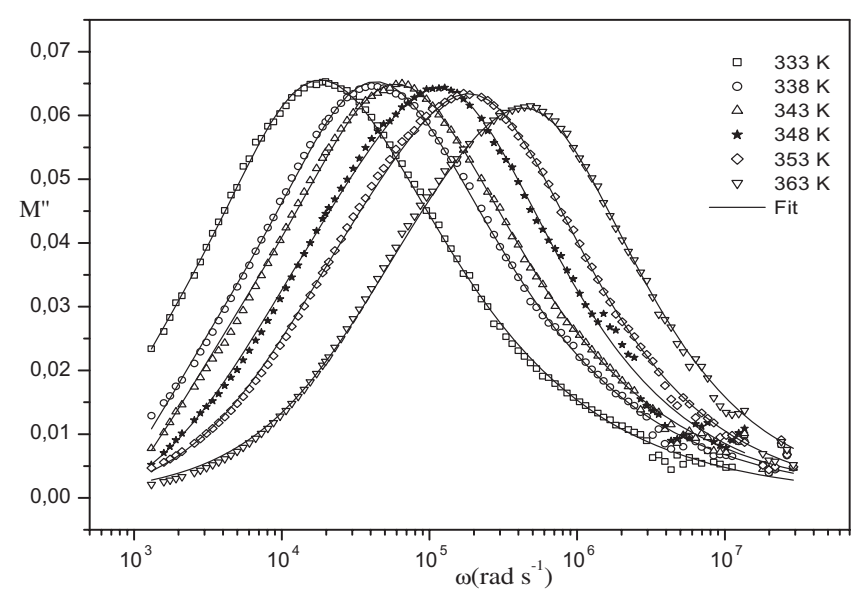

Fig. 11. Variation of imaginary part of electrical modulus $\left(M^{\prime \prime}\right)$ with angular frequency in bis(2-amino-6-methylpyridine) tetrachloridozincate at several temperatures. 
shows the spread of relaxation times with different time constant, and hence relaxation is of non-Debye type. The shape of the spectrum remains constant but the frequency of the modulus maximum, $M_{\max }^{\prime \prime}$ shifts to higher frequency side with increase in temperature. The peak heights at different temperatures are nearly the same. The constancy of the height of the modulus plot suggests the invariance of the dielectric constant and distribution of relaxation times with temperature. ${ }^{38}$ The existence of low frequency peaks suggests that the ions can move over long distances whereas high-frequency peaks suggest confinement of ions in their potential well. The nature of modulus spectrum confirms the existence of hopping mechanism in the electrical conduction of the material.

The modulus plot is nonsymmetric in agreement with the nonexponential behavior of the electrical function, which is well described by the Kohlrausch-William-Watts (KWW) exponential function: ${ }^{39,40}$

$$
\phi(t)=\exp \left(-\left(\frac{t}{\tau}\right)^{\beta}\right) \quad 0<\beta<1
$$

where $\tau$ is the characteristic relaxation time and $\beta$ is the well-known Kohlrausch parameter, which decreases with an increase in the relaxation time distribution. The $\beta$ value is positioned in the range $0<\beta<1$, which represents the deviation from the linear exponential $(\beta=1)$. In the present case, the shape of each spectrum has been quantified with a $\beta$ value obtained by fitting the curve to Eq. (10) by the method of Moynihan et al. ${ }^{41}$ The initial value of $\beta$ was determined from the full width-at-half-maximum of the $M^{\prime \prime}$ spectrum $(\beta=1.14 / \mathrm{FWHM})$ and it was found to be 0.74 . To achieve the fit to Eq. (10) at each temperature, $\beta$ and $M_{\infty}$ values have been taken as freely adjustable parameters. The solid lines through the modulus spectra in Fig. 11 shows the fitted data in good agreement with the experimental data. The $\beta$ value does not change much with the increase in temperature. In the present case, the frequency exponent $\beta$ does not obey Ngai's relation ${ }^{42} \beta=1-n$. The difference arises from the fact that the Kohlrausch function used for fitting the observed modulus spectra leaves the high frequency component unaccounted. In contrast, the conductivity formalism takes into account the high frequency region of the conductivity spectra.

The conductivity relaxation frequency $\omega_{m}$ is given by the relation $\omega_{m}=\omega_{m} \exp \left(E_{a} / k T\right)$ where

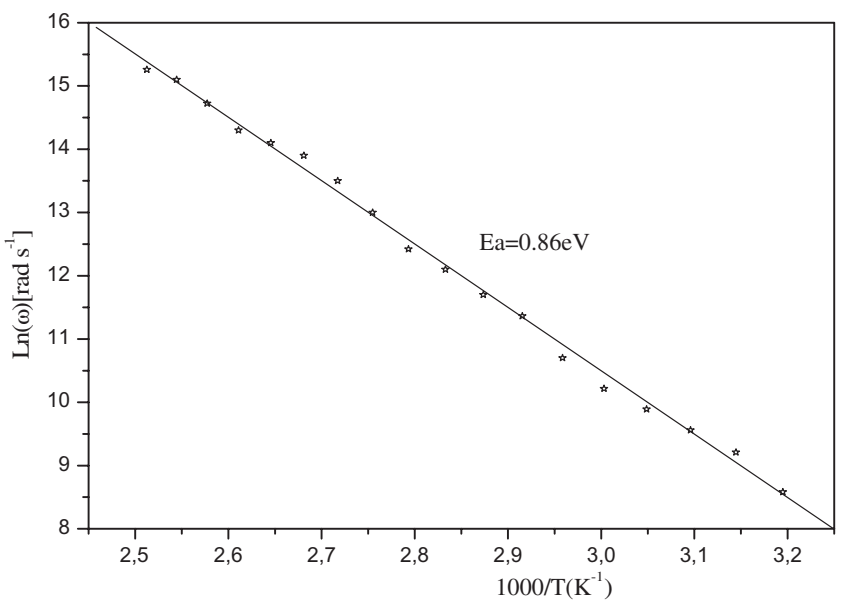

Fig. 12. Inverse temperature dependence of $\operatorname{Ln}(\omega)$ versus reciprocal temperature.

$\omega_{m}$ is the characteristic phonon frequency, $E_{m}$ is the activation energy for conductivity relaxation, $k$ is the Boltzmann constant and $T$ is the temperature. The temperature dependence of the conductivity relaxation frequency is plotted in Fig. 12. It is well described by the Arrhenius relation. The activation energy $E_{m}$ obtained from the modulus spectra is about $0.86 \mathrm{eV}$. The near value of activation energy of both impedance and modulus spectrum suggests that transport of the charge carrier is by a hopping mechanism. ${ }^{43,44}$

\section{Conclusion}

The analysis of the frequency dispersion of the real imaginary components of the complex impedance allowed us to determine an equivalent electrical circuit for the electrochemical cell with bis(2-amino6 -methylpyridine) hexachloridozincate. The stretching exponent $\beta$ is found to be temperature independent with a value of 0.74 . The analysis of the temperature variation of $M^{\prime \prime}$ peak indicates that the observed relaxation process is thermally activated. The near value of activation energies obtained from the analysis of equivalent circuit, modulus and conductivity data confirm that the transport is through ion hopping mechanism in the investigated sample.

\section{References}

1. A. S. Albrecht, C. P. Landee, Z. Slanic and M. M. Turnbull, Molec. Cryst. Liq. 305, 333 (1997). 
2. P. R. Hammar, D. C. Dender, D. H. Reich, A. S. Albrecht and C. P. Landee, J. Appl. Phys. 81, 4615 (1997).

3. G. Meyer and J. Nockeman, Solid State Chem. 162, 254 (2001).

4. P. G. Lacroix, Chem. Mater. 133, 495 (2001).

5. Y. Cui, J. Ren, G. Chen, W. Yu and Y. Qian, Acta Cryst. C 56, 552 (2000).

6. P. G. Lacroix, R. Clement, K. Nakatani, J. Zyss and I. Ledoux, Science 263, 658 (1994).

7. V. Chakravarthy and A. M. Guloy, Chem. Commun., 697 (1997).

8. R. D. Willett, S. F. Haddad and B. Twamley, Acta Crystallogr. Sec. C 56, 437 (2000).

9. B. J. Prince, M. M. Turnbull and R. D. Willett, J. Coord. Chem. 56, 441 (2003).

10. N. Sivron, T. E. Grigereit, J. E. Drumheller, K. Emerson and R. D. Willett, J. Appl. Phys. 75, 5952 (1994).

11. M. R. Bond, R. D. Willett and G. V. Rubenacker, Inorg. Chem. 29, 2713 (1990).

12. F. A. Cotton and A. Yokochi, Inorg. Chem. 37, 2723 (1998).

13. Z. M. Jin, N. Shun, Y. P. Lu, M. L. Hu and L. Shen, Acta Crystallogr. C 61, 43 (2005).

14. M. Nadeem, M. J. Akhtar and A. Y. Khan, Solid. State Commun. 134, 431 (2005).

15. P. Muralidharan, M. Venkateswarlu and N. Satyanarayana, J. Non-Cryst. Solid 351, 583 (2005).

16. J. R. Macdonald, Impedance Spectroscopy Emphasizing Solid Materials and Systems (John Wiley and Sons, New York, 1987).

17. A. Ben Rhaiem, F. Hlel, K. Guidara and M. Gargouri, J. Alloys Compd. 440, 463 (2008).

18. P. S. Anantha and K. Hariharan, J. Phys. Chem. Solids 64, 1131 (2003).

19. V. Hornebecq, J. M. Reau and J. Ravez, Solid State Ionics 127, 231 (2000).

20. H. Mahamoud, B. Louati, F. Hlel and K. Guidara, Ionics 223, 228 (2011).

21. A. K. Jonscher, Nature 264, 673 (1977).

22. B. Louati, M. Gargouri, K. Guidara and T. Mhiri, J. Phys. Chem. Solid 66, 762 (2005).
23. N. Hannachi, I. Chaabane, K. Guidara, A. Bulou and F. Hlel, Mater. Sci. Engrg. B 24, 32 (2010).

24. H. Jain and J. N. Mundy, J. Non-Cryst. Solids 91, 315 (1987).

25. N. F. Mott and E. A. Davis, Electronic Progresses in Non-Crystalline Solids (Clarendon, Oxford, 1970).

26. J. C. Dyre and T. B. Schroder, Rev. Mod. Phys. 72, 873 (2000).

27. S. R. Elliott, Solid State Ionics 27, 131 (1988).

28. A. Ben Rhaiem, N. Zouari, K. Guidara, M. Gargouri and A. Daoud, J. Alloys Compd. 1, 387 (2005).

29. W. K. Lee, J. F. Liu and A. S. Nowick, Phys. Rev. Lett. 67, 1559 (1991).

30. R. G. Palmer, D. L. Stein, E. Abrahams and P. W. Anderson, Phys. Rev. Lett. 53, 958 (1984).

31. P. B. Macedo, C. T. Moynihan and R. Bose, Phys. Chem. Glasses 171, 179 (1972).

32. C. T. Moynihan, L. P. Boesch and N. L. Laberge, Phys. Chem. Glasses 122, 125 (1973).

33. M. Ganguli, M. Harish Bhat and K. J. Rao, Phys. Chem. Glasses 40, 297 (1999).

34. S. Lanfredi, P. S. Saia, R. Lebullenger and A. C. Hernandes, Solid State Ionics 146, 329 (2002).

35. S. Ghosh and A. Ghosh, Solid State Ionics 149, 67 (2002).

36. F. Borsa, D. R. Torgeson, S. W. Martin and H. K. Patel, Phys. Rev. B 46, 795 (1992).

37. B. Louati, K. Guidara and M. Gargouri, J. Alloys Compd. 347, 351 (2009).

38. J. M. Reau, S. Rossignol, B. Tanguy, J. M. Rojo, P. Herrero, R. M. Rojas and J. Sanz, Solid State Ionics 65, 74 (1994).

39. R. Kohlrausch, Progr. Ann. 123, 393 (1847).

40. G. Williams and D. C. Watts, Trans. Faraday Soc. 66, 80 (1970).

41. C. T. Moynihan, L. P. Boesch and N. L. Laberge, Phys. Chem. Glasses 14, 122 (1973).

42. K. L. Ngai, Comments Solid State Phys. 9, 127 (1979).

43. M. Pant, D. K. Kanchan, P. Sharma and M. S. Jayswal, J. Mater. Sci. Engr. B 149, 18 (2008).

44. A. R. West, D. C. Sinclair and N. Hirose, J. Electroceram. 1, 65 (1997). 